Research report

\title{
Oscillatory neuromagnetic activity induced by language and non-language stimuli
}

\author{
Carsten Eulitz ${ }^{\mathrm{a}, \mathrm{c}, *}$, Burkhard Maess ${ }^{\mathrm{b}}$, Christo Pantev ${ }^{\mathrm{c}}$, Angela D. Friederici ${ }^{\mathrm{b}}$, Bernd Feige ${ }^{\mathrm{a}}$, \\ Thomas Elbert ${ }^{\mathrm{a}, \mathrm{c}}$ \\ a Department of Psychology, Postfach 5560-D23, University of Konstanz, Universitätsstraße 10, 78434 Konstanz, Germany \\ ${ }^{\mathrm{b}}$ Max-Plank-Institute for Cognitive Neuroscience, Leipzig, Germany \\ ${ }^{\mathrm{c}}$ Institute for Experimental Audiology, University of Münster, Münster, Germany
}

Accepted 27 February 1996

\begin{abstract}
Event-related oscillatory brain activity during language perception differs from activity occurring during the processing of comparable non-language stimuli. This fact became apparent in the observation of changes in the normalized spectral power of magnetoencephalographic (MEG) signals during the subject's processing of these stimuli. MEG was recorded over the left and right hemispheres of 12 right-handed subjects. During the experimental session, bisyllablic content words and physically similar non-language stimuli were presented with equal probability in a randomized order in either the visual or auditory modality. Approximately $15 \%$ of these stimuli were marked and the subject's task was to detect these marked stimuli. As a major characteristic of language vs. non-language processing, we obtained an enhancement of the normalized spectral power around $240 \mathrm{~ms}$ in the $60-65-\mathrm{Hz}$ band over the left hemisphere for the language condition and over the right hemisphere for the non-language condition, independent of the modality of stimulus presentation. Starting at approximately the same latency but in lower-frequency bands (15-45-Hz), an extended (250-600 ms) reduction of normalized spectral power was observed. This reduction, although it generally confirmed previous results, differed in that no hemisphere-specific reduction was found for the processing of words. A domain-specific enhancement of normalized spectral power was also evident around $800-1200 \mathrm{~ms}$ in the $15-30-\mathrm{Hz}$ band. In the auditory condition, this enhancement of the normalized spectral power was larger after the presentation of language stimuli whereas in the visual condition a larger enhancement of the normalized spectral power was obtained after presentation of non-language stimuli. As this latter effect appears relatively late after the stimulus onset and differs in expression for both modalities of stimulus presentation, a simple relationship between language perception and oscillatory brain dynamics can be excluded for this enhancement. In contrast, the left hemispheric enhancement of the normalized spectral power present around $240 \mathrm{~ms}$ in the $60-65-\mathrm{Hz}$ band seems to reflect oscillatory pattern specific to the processing of words.
\end{abstract}

Keywords: Magnetoencephalography; MEG; Visual evoked magnetic field; Auditory evoked magnetic field; Language; Lexical processing; Cell assembly; Oscillatory brain activity

\section{Introduction}

By using a variety of imaging and measurement techniques, such as Positron Emission Tomography (PET) and functional Magnetic Resonance Imaging (fMRI), which deliver a relatively static view of metabolic changes in activated brain regions [14,33], as well as methods, such as EEG and MEG, functional changes in human brain activity have been repeatedly examined in an attempt to analyse the short term alterations of brain activity which are

\footnotetext{
* Corresponding author. Fax: (49) (7531) 88-2891. E-mail: carsten.eulitz@uni-konstanz.de
}

associated with language processing. Although several ERP components related to language processing have been described, the language specificity of the different ERP components is still under discussion and a search for other parameters indicating this specificity seems to be necessary (for a review, see $[9,10,21,22,31]$ ).

An alternative method of quantifying changes in brain activity is to evaluate brain rhythms, a method which has been used since the beginning of EEG analyses [2] and was recently applied successfully to the evaluation of movement-related activity $[20,36]$ as well as to the investigation of spontaneous brain rhythms, such as $\alpha$ - or murhythm [42], and to the description of processes during 
language perception and production [19,35,25,38]. The spectral composition of EEG and MEG data can be obtained with a comparably high temporal resolution (within limitations given by the 'uncertainty principle'), using short-time spectral analyses [15,27]. Depending on the required frequency band and its bandwidth, the time resolution of this technique is better than hundreds of milliseconds allowing for a successful evaluation of dynamical patterns of brain activity. Moreover, this approach warrants that the brain signals of time- but not phase-looked processes are preserved after averaging across repeated events, thus, revealing aspects of brain activity that are not visible with time-domain analysis.

The neurophysiological plausibility of the evaluation of brain rhythms in different frequency bands is given by a number of experimental findings reviewed by Steriade et al. [46] and Lopes da Silva [23]. The question of whether oscillations in neuronal networks, as reflected in brain waves, are merely epiphenomena or reflect general functions is still under debate. It has been suggested that higher-frequency oscillations $(>20 \mathrm{~Hz})$ may mediate the formation of assemblies of neurons that represent a given stimulus pattern. The activity of such an assembly of neurons or cell assembly [12] is characterized by the coherent firing of large groups of neurons. Experimental evidence for coherent neuronal activity induced by changes in essential features of sensory stimuli comes from local slow wave field-, single- and multi-unit recordings from the olfactory system and the visual system of animals $[4,7,13,44]$. If these coherently activated neurons represent some part of a cortical cell assembly, then cell assemblies can be assumed to be the units by which elementary cortical functions are realized. Thus, one way of exploring these distinct cognitive processes in the human brain could be to measure coherent oscillations with non-invasive techniques, such as EEG or MEG.

For the detectability of cell assembly activity using MEG recordings, the number of coherently activated neurons and their spatial distribution is critical. Previously, only simulation studies were available to estimate the number of neurons belonging to one cell assembly. Aertsen and coworkers [1] recently reported sizes of cell assemblies ranging from a few thousand to several ten thousand neurons, with a fraction of $1 / 10-1 / 5$ of the assembly activated at any given moment (within a 2-4-ms time window). This means in the ideal case that about twenty thousand neurons of one cell assembly could be activated at a time. As pointed out by Wikswo [49], the combined activity of ten to one-hundred thousand neurons is necessary to generate a magnetic field detectable outside the skull. From these considerations, it is clear that only large, not too widely distributed cell assemblies can be detected non-invasively. Given that the estimated number of neurons belonging to one cell assembly is correct, MEG recordings of cell assembly activation may operate near the boundary of detectability. Indeed, in a pattern motion study, Valdes-Sosa et al. [47] did not find an increase in the $\gamma$-band EEG power when bars moved more coherently, as predicted from previous animal studies reviewed by Singer [44]. One explanation may be that stimulation included a full visual field and that a part of the induced oscillatory brain activity was canceled out for neuroanatomical reasons. Comparable studies presenting relevant stimuli in a single visual hemifield were able to detect induced $\gamma$-band responses over the occipital brain regions $[26,29]$ illustrating that not only the number of neurons belonging to one cell assembly is important but also their spatial arrangement.

Based on Braitenberg and Pulvermüller's [3] 'neurological theory of language' (see also [37]), it is assumed that large cell assemblies are activated during the perception of linguistic elements, such as words. This theory maintains that the presentation of words to an active listener leads to a subsequent activation of specific cortical cell assemblies which results in a fast, periodic, coordinated activation of a large number of neurons. Pulvermüller et al. [38] and Lutzenberger et al. [25] go on to suggest that this periodic activity can be quantified by calculating the spectral power changes as compared to a baseline level in the frequency bands of $>20 \mathrm{~Hz}$. It is maintained that word perception should lead to a cell assembly ignition and, thus, enhance the spectral power compared to the baseline. In contrast, it is theorized that the perception of stimuli, such as non-words or non-language stimuli similar to those applied in the present study, are not expected to activate large and strongly coupled cell assemblies and that no change in the spectral power with respect to the baseline should follow.

In the present study, language and non-language stimuli which were matched for physical structure and complexity were used. In order to test the assumption that different input modalities initiate the same neuronal subsystems responsible for language processing [16,30,43], stimuli were delivered both in the auditory and visual modality. If this assumption holds true, a similar topography of language-related effects can be expected for both modalities of stimulus presentation but not for effects obtained with the non-language stimuli.

One goal of this study was to quantify stimulus-induced changes in the oscillatory brain activity and to analyse whether or not these changes were different for the perception of language compared to non-language stimuli. In addition, the study aimed to examine the dependence of these changes on the modality of stimulus presentation and, if possible, to analyse the topographical aspects and hemispheric lateralization of these changes. One final goal was to examine whether or not the changes in oscillatory brain activity could be explained by an activity of wordspecific cell assemblies, as suggested in the 'neurological theory of language' [3]. In this case, one would expect to see an enhancement of spectral power in the $\gamma$-band in response to the auditory and visual presentation of lan- 
guage stimuli over brain regions relevant for language processing. In contrast, the non-language stimuli should induce topographically different alterations in $\gamma$-band activity.

\section{Materials and methods}

\subsection{Subjects}

Twelve right-handed subjects (six female and six male) aged 22-32 years (median age 26.5 years) participated in the experiment. Handedness was determined using the Edinburgh handedness questionnaire [32]. Two subjects were excluded from further analyses who had a laterality score $<90 \%$ as well as a high artifact rejection ratio. The mean laterality score across the remaining 10 subjects was 100. None of their close family members (parents, siblings) were left-handed.

\subsection{Stimuli and procedure}

42 German words belonging to the lexical class of content words and 42 different non-language stimuli were used for presentation. An equal number of blocks with stimuli either in the visual or the auditory modality were delivered. The experiment consisted of four recording blocks (auditory and visual stimulation respectively, for the left and the right hemisphere). The order of blocks was balanced across subjects with the constraint that half of the subjects were measured over the left hemisphere first and that half of these subjects started with the auditory modality. For each subject, a total of 252 stimuli/experimental condition were delivered.

Words were bisyllablic, with each syllable following a consonant-vowel pattern. The consonants of the first syllable were all plosives $(/ \mathrm{b} /, / \mathrm{p} /, / \mathrm{d} /, / \mathrm{t} /, / \mathrm{g} /, / \mathrm{k} /)$. Non-language stimuli were derived from the language stimuli by modification of their physical characteristics.

In the visual condition, stimuli consisted of four black characters on a light grey background. Words were written using the Geneva font; the non-language stimuli consisted of false font characters (see Fig. 1). Visual stimuli were presented for $400 \mathrm{~ms}$ by means of a non-magnetic stimulus delivery system based on a fiber optic. The interstimulus interval was varied randomly from 1.8 to $2.2 \mathrm{~s}$. The screen was positioned $60-70 \mathrm{~cm}$ from the subject's eyes. During the measurement, a frame (visual angles: $3.1^{\circ}$ vertical, $6.8^{\circ}$ horizontal) was displayed continuously on the screen and subjects were asked to fixate their gaze on the middle of the frame. Stimuli were presented in the centre of the frame, with the maximum size being $1.4^{\circ}$ vertical and $4.1^{\circ}$ horizontal.

For the auditory blocks, words were spoken by a professional female speaker and digitally recorded. In order to make them physically similar, stimuli were equalized in

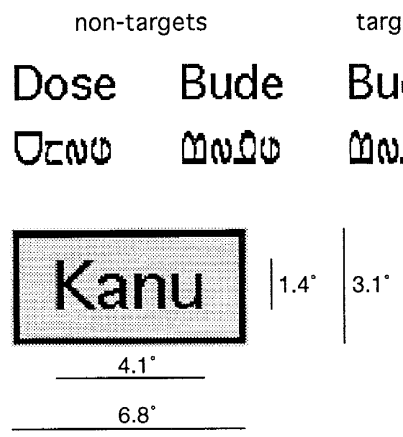

Fig. 1. Examples of visual language- and non-language stimuli out of a set of 42 different variants /stimulus class. Target stimuli were derived from the non-target stimuli by cutting out a small rectangle of one of the characters. In the bottom part of the figure, a visual language stimulus as it was delivered during the experimental session is shown. Respective visual angles are indicated.

duration and as much as possible in their onset characteristics. The non-language stimuli were the spoken words played backwards. The binaurally presented auditory stimuli (75-80 dB SL) had a duration of $570 \mathrm{~ms}$ and were delivered using an echo-free non-magnetic stimulus delivery system with the same pause between stimulus presentations as in the visual condition.

In creating the subject's task the following goals were taken into consideration: (a) identical attentional level to both classes of stimuli; (b) no impact of movement-related brain activity on relevant epochs due to responses; and (c) restriction to lower-order linguistic processes, such as word perception. The first two points were realized by using a target detection task for which targets were recruited from both stimulus classes, similarly manipulated, and presented with equal probability. In order to satisfy point c, subjects simply attended language and non-language stimuli without performing any specific linguistic task. As suggested by experimental results on the 'word superiority effect' $[40,48]$, meaningful words are processed differently than non-words even when not required by the experimental procedure.

Target stimuli were inserted into the sequence of language and non-language stimuli occurring with a probability of $15 \%$. The targets were identical to the set of stimuli described above, with the exception that for the visual stimuli a small rectangle was cut out with an area of approximately $40 \%$ of one of the characters (Fig. 1) and that for the auditory modality $60 \mathrm{~ms}$ of the original stimuli were substituted by a two-tone sound (880 and $1109 \mathrm{~Hz}$ ) starting randomly at $100-400 \mathrm{~ms}$ after stimulus onset. The subject's task was to detect the targets and to respond by pressing a button as quickly and accurately as possible. The presentation of language- and non-language- targets was equiprobable and the subjects had no language vs. non-language decisions to make in the course of the experimental session. The monitoring task was used to focus the subject's attention to the stimuli. Because there 
were relatively few targets for this task, brain responses to these stimuli were not analysed.

During an instructional session prior to the experiment, all participants were able to identify all words and target stimuli with an accuracy of $100 \%$. During the preparation for the measurement and during the instructional session, the stimuli were repeatedly presented to the subjects in order to prevent repetition effects to occur later in the experiment.

\subsection{MEG measurements}

Neuromagnetic data were recorded using a 37-channel neuromagnetometer (Magnes, Biomagnetic Technologies; consisting of first-order axial gradiometers with 5-cm baseline; pick-up coils are arranged in an array of concentric circles with a diameter of $14.4 \mathrm{~cm}$ ). Measurements were carried out in a magnetically shielded room. The sensor array was centred sequentially over the left and the right supratemporal cortex about $1.5 \mathrm{~cm}$ superior to the T3 or the T4 electrode position of the 10-20 system, respectively. The head position relative to sensor pickup coils was measured by a sensor position indicator. The horizontal and vertical EOG and the button press were also registered. Subjects were lying on their side with their head fixated by a vacuum cushion. They were instructed to avoid eye blinks and head movements and to carry out the monitoring task carefully. Continuous data were recorded in 13-min blocks at a sampling rate of $297.6 \mathrm{~Hz}$ with a passband of $0.03-100 \mathrm{~Hz}$.

\subsection{Data analysis}

Averaged waveforms were calculated for each hemisphere and modality of stimulus presentation selectively for the language and the non-language condition. Button press-free epochs of $1500 \mathrm{~ms}$, including a 300-ms prestimulus baseline, were extracted from the continuous data. Artifact-contaminated epochs with a signal deviation of $>2.5 \mathrm{pT}$ in the MEG and $120 \mu \mathrm{V}$ in the EOG were excluded from the mean values. The averaged waveforms were digitally filtered using a low-pass filter of $20 \mathrm{~Hz}(12$ $\mathrm{dB} /$ oct).

For frequency domain analyses, a method similar to the event-related perturbation analyses [27] was applied. In epochs of artifact-free raw data (same rejection criteria as reported above), power spectral estimates were derived from Fourier transforms on pairs (overlapping by one half) of 107-ms Welch-windowed segments, logarithmized and selectively averaged for each segment around stimulus onset and experimental condition, i.e. modality of stimulus presentation, hemisphere of measurement and stimulus class. Nine time segments were equidistantly situated within a 600-ms interval before stimulus onset (baseline) and 21 segments after stimulus onset within an interval of $1200 \mathrm{~ms}$. After averaging, the exponential was calculated to yield spectral power estimates. Only spectral power changes in frequency bands of $>7 \mathrm{~Hz}$ were considered. Under these constraints, a frequency resolution of $4.6 \mathrm{~Hz}$ was obtained. In order to examine whether or not the observed high-frequency effects are in part an epiphenomenon of equivalent changes in the $\alpha$-band [17], the full power spectrum was evaluated.

The mean power spectra were transformed with respect to baseline-related changes in two different ways. First, normalized mean power spectra were calculated by dividing each singular mean spectral power within one frequency bin by the mean spectral power across all corresponding baseline segments. A second parameter was the portion of single trial spectral power estimates below or above the mean single trial baseline spectral power (relative gain). This parameter was obtained by comparing the spectral power for a window position with the respective baseline values separately for all experimental conditions and time/frequency bins. This relative gain is insensitive to occasional deviant spectral power estimates and was used to verify differences occurring in normalized spectral power.

In order to further reduce the data and to obtain estimates that are not affected by small alterations in the location or orientation of the generating brain regions and that show little dependency on individual variations of brain anatomy, the normalized spectral power and the relative gain were collapsed across all 37 channels above one hemisphere. This led to a smoothing and to a reduction of effects when significant changes in spectral power were only local. Furthermore, topographical information was reduced to hemispheric differences. However, the mean differences between experimental conditions were small and it was preferred to use robust parameters and conservative statistical testing. For a more detailed topographical analysis of effects found with the conservatively reduced data, three submean values of 12 magnetic channels were

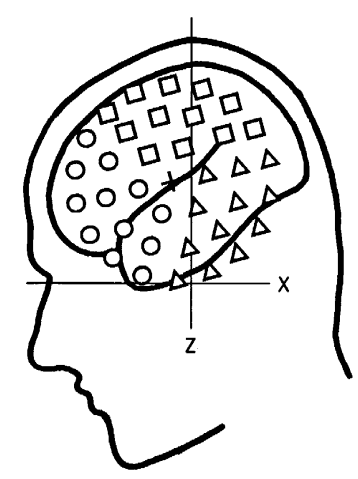

Fig. 2. Projection of the 37 channels of the used MEG device on a left hemispheric scheme of a head. Different symbols indicate the channels used for submean values of normalized spectral power for more detailed topographical analyses. Circles represent the frontotemporal, rectangles the centroparietal and triangles the temporooccipital brain regions. Axes demonstrate approximate locations of the posterior-anterior $(\mathrm{x})$ and inferior-superior directed axes of a head-centred coordinate system. 
calculated. The averaged channels were situated over the frontotemporal, the centroparietal and the temporooccipital brain regions. Across subjects, the brain regions were aligned with the middle channel of the sensor array which was positioned $1.5 \mathrm{~cm}$ superior above the $\mathrm{T} 3$ or the $\mathrm{T} 4$ electrode position in all subjects and with the most superior channel of the sensor array (see Fig. 2).

The normalized spectral power and the relative gain were statistically tested as dependent variables for differences using three-way univariate ANOVAs for a 2-MODALITIES (auditory vs. visual) $\times 2$ STIMULUS CLASSES (language vs. non-language) $\times 2$ HEMISPHERES (left vs. right) design (repeated measures). ANOVAs were carried out for time/frequency bins where the grand mean of the normalized spectral power across all subjects exceeded the baseline level by $1 \%$ in at least one of the experimental conditions. Effects were reported only if both the normalized spectral power and the relative gain indicated statistical significance $(P<0.05)$.

\section{Results}

\subsection{Performance data}

In the auditory condition, the mean reaction time to target stimuli was $778 \mathrm{~ms}$ (the mean onset time of the target maker was $270 \mathrm{~ms}$ after stimulus onset) and the mean error rate was $5 \%$. Parameters showed no difference between the language and the non-language condition. In

left hemisphere

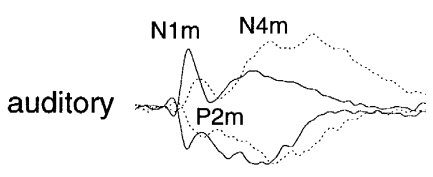

language non-language

visual
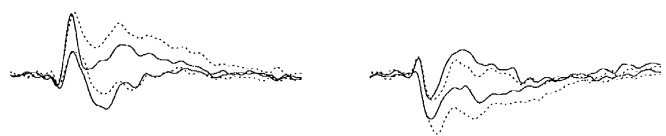

$100 \mathrm{f}$
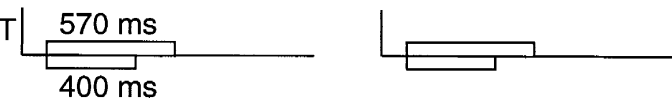

Fig. 3. Grand mean event-related field waveforms across all subjects for all experimental conditions measured above the left and the right hemisphere are seen here. The two channels with maximum ingoing and outgoing magnetic fields in the latency range of $400-600 \mathrm{~ms}$ of the auditory language condition were chosen for each subject and were then taken for the other experimental conditions. The dotted line represents the non-language condition; the solid line the language condition. The onand off-set as well as the duration of stimuli in the two modalities of stimulus presentation are indicated in the bottom part of the figure. Only $100 \mathrm{~ms}$ of the $300-\mathrm{ms}$ baseline were plotted.

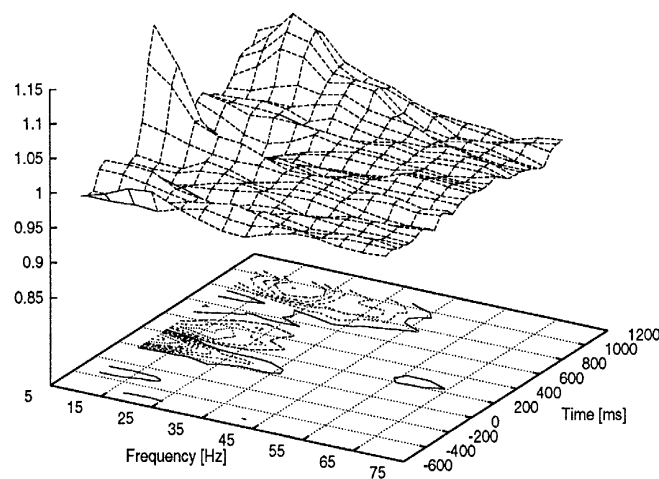

Fig. 4. This figure illustrates for one experimental condition how the isocontour plots used in Fig. 5 were derived from the normalized spectral power values of all time/frequency bins. The $x$-axis represents the frequency, the $y$-axis the latency and the $z$-axis the normalized spectral power. The surface plot indicates the changes in normalized spectral power in the time-frequency plane. The same information is illustrated in the bottom part using an isocontour plot with a contour step of $1.5 \%$. A normalized spectral power of 1 corresponds to the mean spectral power across nine prestimulus time windows (separately calculated for each frequency band). A normalized spectral power of 1.06 indicates their enhancement by $6 \%$ above baseline level.

the visual condition, the mean reaction time to target stimuli in the language condition was $617 \mathrm{~ms}$ with a mean error rate of $3 \%$ but $536 \mathrm{~ms}$ to non-language targets with a mean error rate of $16 \%$. The influence of the STIMULUS CLASS on both the reaction time and the error rate in the visual condition was statistically significant $(P<0.001)$.

\subsection{Waveforms}

In order to determine general data quality as well as consistency of results with other EEG and MEG studies, signals for two channels were separately grand averaged across all subjects under all experimental conditions. Results are shown in Fig. 3. The two channels chosen for each subject were the channels with the maximum ingoing and outgoing magnetic field in the latency range of 400$600 \mathrm{~ms}$ of the auditory language condition. The same channels were then taken for the other experimental conditions. The first prominent component in the auditory evoked field is the N1m, which is suppressed in the non-language condition. Around 400-600 ms, a high amplitude component occurs which has their ingoing and outgoing magnetic fields at approximately the same sensors as the $\mathrm{N} 1 \mathrm{~m}$ in the auditory modality. This component is present for all experimental conditions and seems to correspond to the magnetic N400 (N4m). Its amplitude is more pronounced for the non-language condition as compared to the language condition, independent of presentation modality or hemisphere ${ }^{1}$.

\footnotetext{
${ }^{1}$ Detailed analyses of the component characteristics, such as their amplitude and source parameters, will be reported in a forthcoming publication.
} 


\subsection{Averaged normalized spectral power}

Changes of the normalized spectral power averaged across subjects in all time/frequency windows for all experimental conditions are illustrated (for a detailed de- scription of how to interpret the isocontour plots, see Fig. 4) in Fig. 5 .

There are two major time/frequency regions where the spectral power is enhanced compared to the baseline and one time/frequency region showing a reduction of spectral

auditory stimuli

left hemisphere

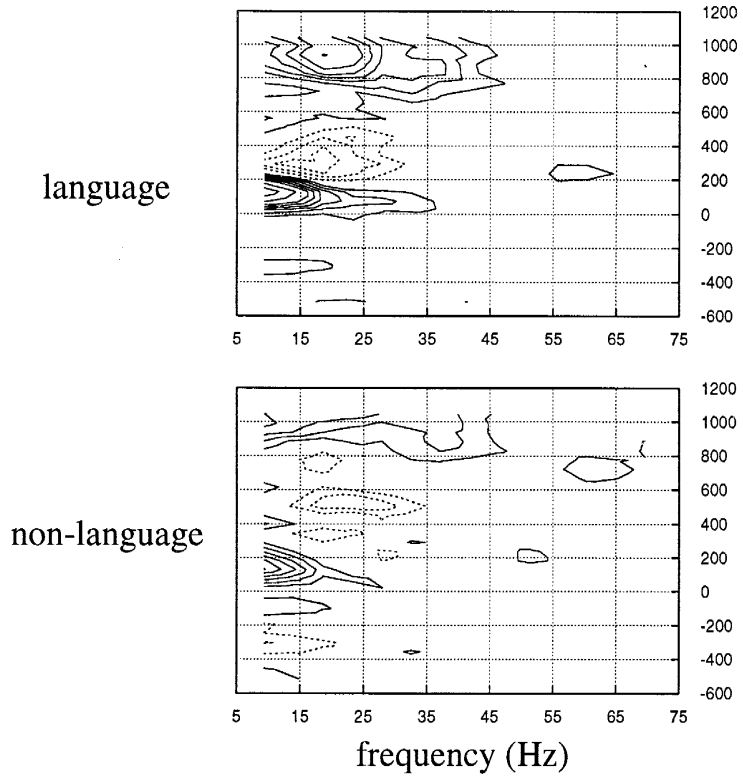

right hemisphere
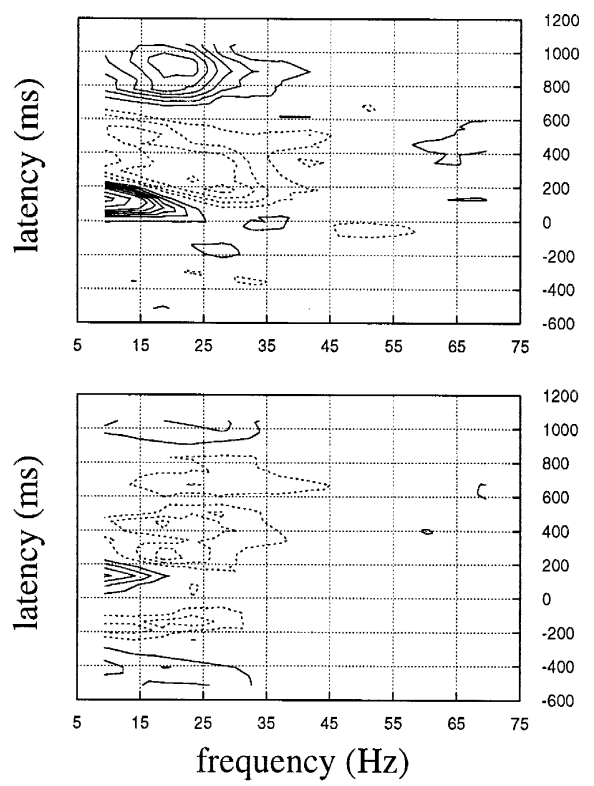

visual stimuli
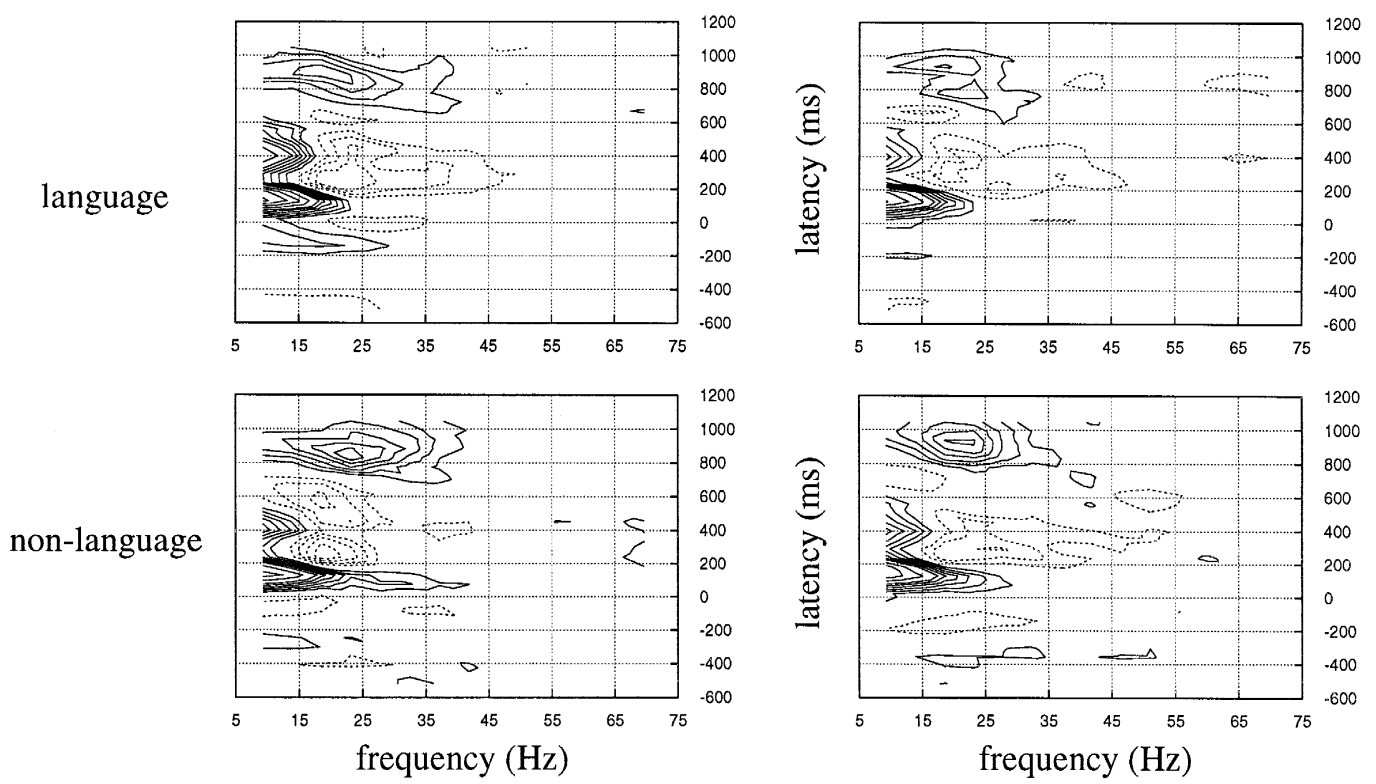

Fig. 5. Normalized spectral power for all time/frequency bins averaged across all channels and then across all subjects is presented here, for all experimental conditions. The left and right parts of the figure illustrate the results from the left and the right hemisphere, respectively. The upper part of the figure displays the results from the auditory condition and the lower part that from the visual condition. In each picture, the $x$-axis represents the frequency, the $y$-axis the latency and the level of normalized spectral power is indicated by isocontour lines. Solid lines used for time/frequency areas of enhanced normalized spectral power by $>1.5 \%$; dotted lines indicate a reduction of normalized spectral power by $>1.5 \%$. The contour step is $\pm 1.5 \%$ up to $\pm 9 \%$ and then $\pm 3 \%$. 
power in most of the experimental conditions. Regions of enhanced spectral power occur around $129 \mathrm{~ms}$ in the 9.3-18.6- $\mathrm{Hz}$ bands and around $883 \mathrm{~ms}$ in the $13.9-32.5-\mathrm{Hz}$ bands. It is easy to see that the enhancement at $129 \mathrm{~ms}$ is less pronounced during the perception of auditory non-language stimuli ${ }^{2}$ whereas the enhancement around $883 \mathrm{~ms}$ depends greatly on the modality of stimulus presentation. In the auditory condition, the enhancement at $883 \mathrm{~ms}$ is larger during the perception of language stimuli whereas in the visual condition non-language stimuli leads to a more increased enhancement of the normalized spectral power. A reduction of normalized spectral power is seen around $345 \mathrm{~ms}$ in the $13.9-41.8-\mathrm{Hz}$ range. This reduction and the enhancement of normalized spectral power around $883 \mathrm{~ms}$ have their maximum deviations from the baseline level in approximately the same frequency bands around $20 \mathrm{~Hz}$ and seemed to be related, sequentially occurring effects which take place in response to the task. A further enhancement of normalized spectral power in frequency bands of $<15 \mathrm{~Hz}$ around $400 \mathrm{~ms}$ was only evident in the visual condition. An extended view of the normalized spectral power changes for the $18.6-$ and $60.3-\mathrm{Hz}$ frequency bands as a function of time is presented in Fig. 6.

Spectral power estimates were derived from 161-ms time segments. Due to this temporal uncertainty, we did not have to postulate an absolute synchrony of the stimulus processing of the two modalities, making statistical testing within every time window reasonable. Statistically significant effects were mostly obtained in several adjacent time/frequency windows. Detailed results, however, will be reported for the time/frequency windows with the maximum deviations of normalized spectral power from the baseline level if both the spectral power and the relative gain indicated statistical significance. The latency and frequency values reported in the following text indicate the middle latency as well as the middle frequency of the corresponding $161-\mathrm{ms}$ time and $4.6-\mathrm{Hz}$ frequency windows.

A statistically significant STIMULUS CLASS by HEMISPHERE interaction $\left(F_{1 / 9}=7.42 ; P<0.03\right)$ was found in the time window centred at $237 \mathrm{~ms}$ in the $60.3 \mathrm{-Hz}$ and $65.0-\mathrm{Hz}$ bands. As seen in Fig. 7, the mean normalized spectral power was enhanced over the left hemisphere for the language condition $(+1.7 \%)$ and over the right hemisphere for the non-language condition $(+1.5 \%)$ during both modalities of stimulation. Changes in the spectral power for the respectively opposite hemispheres did not exceed a $1 \%$ interval around the baseline. For a more detailed topographical analysis of this effect, submean

\footnotetext{
${ }^{2}$ Changes in spectral characteristics of this time/frequency bin were similar to the amplitude differences of ERF components in that latency range. Effects are mainly explained by differences in onset characteristics of the language and non-language stimuli and will not be reported in detail.
}
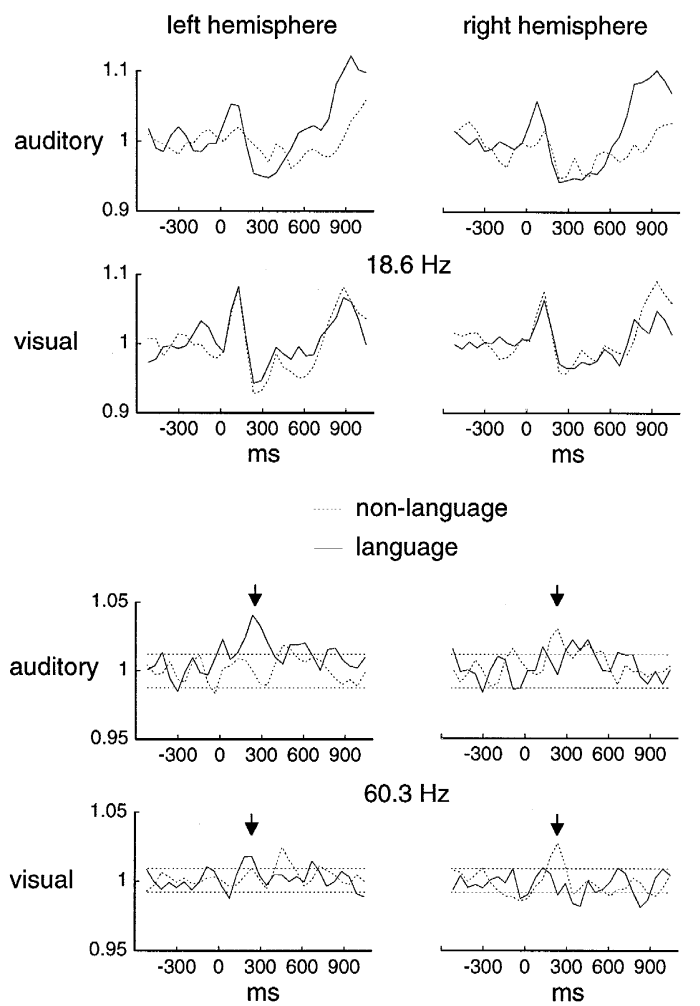

Fig. 6. Waveforms demonstrate the normalized spectral power averaged across all channels and then across all subjects as a function of time for the frequency band around $18.6 \mathrm{~Hz}$ for all experimental conditions (upper panel). At the lower panel, the frequency band around $60.3 \mathrm{~Hz}$ is shown. Here, the spectral power was averaged across centroparietal brain regions for the auditory modality and across temporooccipital brain regions for the visual modality. The dotted line represents the non-language condition; the solid line indicates the language condition.

values of the normalized spectral power from channels over the frontotemporal, the centroparietal and the temporooccipital brain regions were calculated. Mean values of the normalized spectral power are shown in Fig. 7 for all experimental conditions and brain regions. These mean values indicate that the right hemispheric enhancement of the normalized spectral power for the non-language condition is well-expressed over the centroparietal brain region for both modalities of stimulus presentation whereas the left hemispheric enhancement of normalized spectral power for the language condition is situated over the temporooccipital brain region for both the auditory and the visual modality. The largest left hemispheric enhancement of the normalized spectral power for the language condition is seen for the auditory condition over the centroparietal brain region. Statistical analyses using the BRAIN REGIONS (three levels) as an additional repeated measures factor revealed on top of the STIMULUS CLASS by HEMISPHERE interaction $\left(F_{1 / 9}=9.51 ; P<0.02\right)$ a significant main effect of the BRAIN REGIONS $\left(F_{2 / 14}=4.44 ; P<0.04\right)$. The BRAIN REGIONS did not, however, significantly interact with other repeated measures factors. Posthoc Scheffé comparisons suggested that the main effect was mostly caused by 


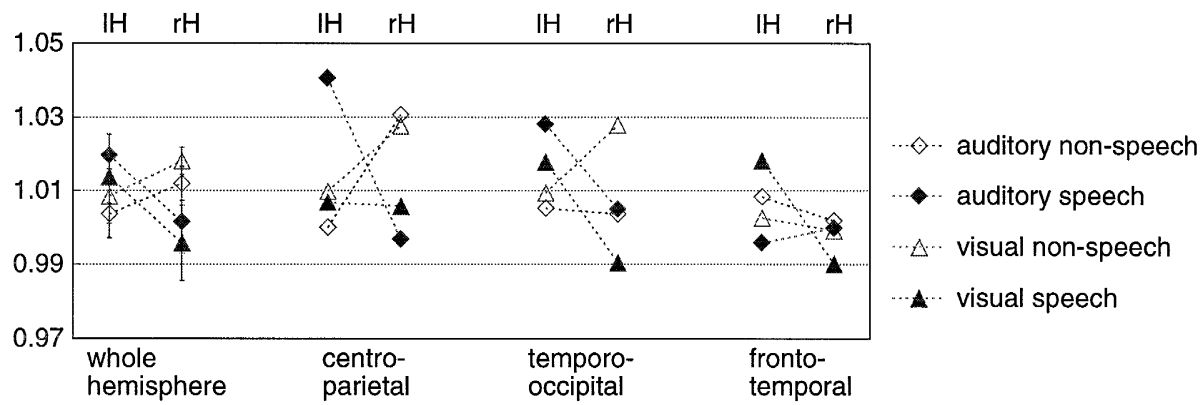

Fig. 7. Normalized spectral power averaged across all channels (left), or across three brain regions (right) and then across subjects separately for all experimental conditions is displayed here for the $237-\mathrm{ms}$ time and $60.3-\mathrm{Hz}$ frequency bin. Below the diagram, the middle latency and frequency of the selected time/frequency bin as well as the corresponding brain region is indicated. Filled symbols represent the language condition, empty symbols the non-language condition. The auditory condition is symbolized by diamonds, the visual condition by triangles. Corresponding parameters from the left and the right hemisphere are connected by a dotted line. Error bars indicate between-subject variability (S.E. values).

differences between centroparietal and frontotemporal brain regions $(P<0.04)$.

Approximately at the same latency, a well-pronounced reduction of normalized spectral power begins which is largest in the $18.6-\mathrm{Hz}$ frequency and $237-\mathrm{ms}$ time bin. As indicated in Fig. 8, the mean reduction of normalized spectral power was larger for the auditory language stimuli than for the corresponding non-language stimuli. The opposite was true for the visual condition. The ANOVA revealed a significant STIMULUS CLASS by MODALITY interaction $\left(F_{1 / 9}=7.21 ; P<0.03\right)$. It can be seen in Fig. 8 that for the auditory condition, the reduction of the normalized spectral power is larger over the right hemisphere as compared to the left hemisphere whereas the visual condition produced a larger reduction in the left hemisphere. The opposite effect can then be observed for the non-language condition. The ANOVA yielded a statistically significant MODALITY by HEMISPHERE interaction $\left(F_{1 / 9}=8.93\right.$; $P<0.02$ ).

For the reduction in normalized spectral power, a modality-independent statistically significant effect of the STIMULUS CLASS could also be observed. This was evident in time/frequency bins outside the maximum reduction of normalized spectral power, viz. in the frequency bands 37.1 and $41.8 \mathrm{~Hz}$, for the time window centred at $291 \mathrm{~ms}$

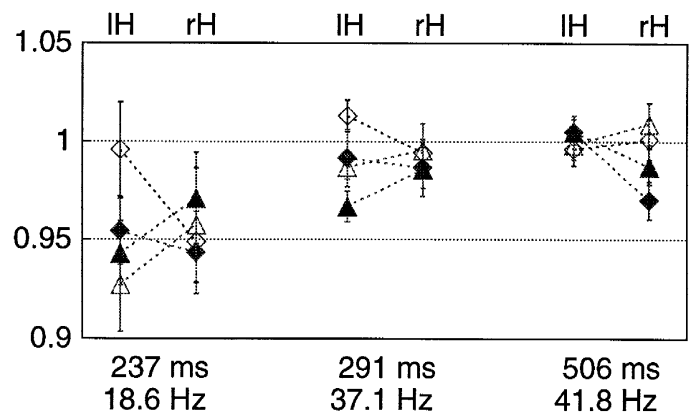

Fig. 8. Normalized spectral power averaged across all channels and subjects for all experimental conditions is displayed here for three time/frequency bins. The middle latency and frequency of the selected time/frequency bins indicating a reduction in normalized spectral power is shown below the diagram (for symbols, see Fig. 7) as well as for the $41.8-\mathrm{Hz}$ band at $345 \mathrm{~ms}$. The main effect of STIMULUS CLASS was most powerful for $37.1-\mathrm{Hz}$ band $\left(F_{1 / 9}=11.08 ; P<0.009\right)$. Independent of hemisphere and stimulus modality, normalized spectral power was more reduced compared to the baseline during the perception of language stimuli $(-1.7 \%)$ than in the non-language condition $(-0.2 \%)$. The differences in mean normalized spectral power between experimental conditions are illustrated in Fig. 8.

In the time window centred at $506 \mathrm{~ms}$ at $41.8-\mathrm{Hz}$ band, the ANOVA revealed a statistically significant STIMULUS CLASS by HEMISPHERE interaction $\left(F_{1 / 9}=12.53 ; \quad P<\right.$ 0.007). Main effects of STIMULUS CLASS $\left(F_{1 / 9}=3.66\right)$ and HEMISPHERE $\left(F_{1 / 9}=4.17\right)$ did not reach the significance level $(P<0.09)$. Whereas the changes in spectral power for the non-language condition as well as for both stimulus classes over the left hemisphere did not exceed a $1 \%$ interval around the baseline, a mean reduction of normalized spectral power of $2.1 \%$ across both modalities was found over the right hemisphere. Posthoc Scheffé comparisons suggested that the interaction originated mainly from language non-language differences in the right hemisphere $(P<0.03)$ and by interhemispheric differences in the language condition $(P<0.03)$.

Figs. 5 and 6 demonstrate the pronounced enhancement of normalized spectral power compared to the baseline around $883 \mathrm{~ms}$ in the $18.6-32.5-\mathrm{Hz}$ bands and its interaction with the modality of stimulus presentation. For example, it can be seen here that the enhancement in the language condition is larger for the auditory modality but lower for the visual modality. The largest enhancement of normalized spectral power was obtained at the $937-\mathrm{ms}$ time and $18.6-\mathrm{Hz}$ frequency window. The ANOVA revealed a statistically significant STIMULUS CLASS by MODALITY interaction $\left(F_{1 / 9}=8.50 ; P<0.02\right)$.

\section{Discussion}

Performance data indicated that the subject's attention to the analysed non-target stimuli was adequate. Auditory 
and visual presentation of language and non-language stimuli elicited a characteristic pattern of changes in normalized spectral power (and of the relative gain), which was modulated by independent variables, such as STIMULus CLASS, and HEMISPHERE, but also by the MODALITY of stimulus presentation. Among all modality-independent effects of the STIMULUS CLASS, only the effect in the $60-\mathrm{Hz}$ band indicated the activation of brain structures specific for linguistic processing in relevant brain structures. This higher frequency effect was obtained around $237 \mathrm{~ms}$ in the 60.3- and $65.0-\mathrm{Hz}$ bands and showed a left hemispheric enhancement of normalized spectral power during the processing of the language stimuli clearly pronounced for both modalities over temporooccipital scalp regions, i.e. in the vicinity of Wernicke's area. The right hemispheric enhancement of normalized spectral power occurred during the processing of non-language stimuli and was best expressed over centroparietal brain areas. Meanwhile, the normalized spectral powers over the corresponding opposite hemisphere remained near the baseline level. This result matches the predictions based on a cell assembly theory of language [3], i.e. word presentation should lead to a cell assembly ignition, because an adequate stimulus has been perceived, while presentation of non-language stimuli should fail to ignite specific assemblies in the same brain regions. Gamma-band responses $(>25 \mathrm{~Hz}$ ) to words should be stronger than responses to non-language stimuli over brain regions processing the words. This hold true for the present results which indicate an enhancement of the $\gamma$-band spectral power over the left hemisphere. As all subjects were right-handed, one could expect that speech would be predominantly processed in this region [24,39].

From a general point of view, our $60-\mathrm{Hz}$ result support the thesis that language is processed predominantly in the left hemisphere $[5,18,28]$ but does not exclude the possibility that a considerable fraction of right hemispheric neurons were activated by the word stimuli [50]. A possible explanation of our hemispheric difference is that the fraction of right hemispheric neurons involved in the cell assembly is too small to be detected with the applied measurement techniques. Evidence for such interhemispheric asymmetry of neuronal activity stems from PET and fMRI studies $[8,14,28,33]$. The results for the non-language stimuli indicated a predominant activation of brain regions in the right hemisphere, as assumed for the processing of visual-spatial information $[11,41,45]$.

The interpretation of spectral power changes in highfrequency bands is usually hampered by a possible influence of muscular activity which has its spectral power maximum around $70 \mathrm{~Hz}$ [6]. The presently measured normalized spectral power peaked $<70 \mathrm{~Hz}$. The topographical differences of normalized spectral power within hemispheres are not consistent with the distribution of muscular activity.

Two other higher-frequency effects indicating modality-independent differences in the perceptual pro- cesses for language and non-language stimuli are characterized by a reduction of the normalized spectral power compared to the baseline level. (1) Normalized spectral power was more reduced around $291 \mathrm{~ms}$ in the 37.1- and $41.8-\mathrm{Hz}$ bands during the perception of language stimuli as compared to non-language stimuli, independent of modality for both hemispheres. Therefore, this reduction cannot be explained as being due to the involvement of hemisphere-specific brain structures of language processing. (2) Normalized spectral power was remarkable lower around $506 \mathrm{~ms}$ in the $41.8 \mathrm{-Hz}$ band during the perception of language stimuli as compared to non-language stimuli in the language-non-dominant right hemisphere for both modalities of stimulus presentation. In the left hemisphere, normalized spectral power was slightly higher during the perception of language stimuli as compared to non-language stimuli. The absolute level, however, was found to be near the baseline.

The results around the $40-\mathrm{Hz}$ band were obtained on the border of the time/frequency area of reduction of normalized spectral power. The largest reduction of normalized spectral power, however, was found in the $15-25-\mathrm{Hz}$ band, where the pattern of changes indicated an influence of the modality of stimulation. This implies that linguistically identical stimuli induced different changes in normalized spectral power depending on whether the language was presented orally or visually. This result is incompatible with the idea that common neuronal subsystems responsible for language processing are initiated by language stimuli delivered via different input modalities [16,30,43]. Therefore, it may be concluded that the pronounced reduction of normalized spectral power in the $15-25-\mathrm{Hz}$ band is not a direct correlate of linguistic processing. In fact, this reduction may be viewed as an event-related desynchronisation phenomenon, i.e. an electrophysiological correlate of cortical activation occurring during stimulus processing, motor output, motor preparation or preparation to process sensory information [34]. According to this view, the reduction in normalized spectral power observed here may be interpreted as a correlate of a sensory modality-specific cortical activation but not of linguistic processing.

The general effect found in the present study, viz. the reduction of normalized spectral power around 300-600 $\mathrm{ms}$, is in agreement with those results reported by Pulvermüller et al. [38] and Lutzenberger et al. [25]. However, upon examining the finer structure of the previous study's results and interpretations notable differences tend to arise in comparison to the present study. For example, in the above-mentioned studies, a reduction of spectral power compared to a baseline for a pseudoword condition was measured over the language-dominant left hemisphere in the latency range of 300-600 ms whereas the spectral power in the $25-35-\mathrm{Hz}$ band did not change in response to word presentation. The authors explained these results, shown in two separate experiments using EEG technique and visual stimuli as well as MEG signals and auditory 
stimuli, as being in accordance with the model of Braitenberg and Pulvermüller [3]. At first, these results appear to be contradictory to predictions based on the 'neurological theory of language' but the results can be explained on the basis of this theory if a long-lasting ignition of wordspecific cell assemblies is assumed influencing prestimulus levels. This assumption seems reasonable given that in these two studies subjects carried out a lexical decision as well as a memory task during the experimental session.

In contrast to the previously cited studies, the present experiment required that subjects detect target stimuli while perceiving language and non-language stimuli. The greatest reduction of spectral power compared to baseline was measured in a lower-frequency band (around $20 \mathrm{~Hz}$ ) but the difference between the language and non-language condition was reversed for the auditory and the visual condition. On the border of the time/frequency area of reduction of normalized spectral power (around $40 \mathrm{~Hz}$ ), we found a more pronounced reduction for the language condition over both hemispheres (around $291 \mathrm{~ms}$ ) as well as predominantly over the right hemisphere (around $506 \mathrm{~ms}$ ), i.e. the pattern of reduction of normalized spectral power did not point to cortical structures in the language-dominant hemisphere. For the time being, the specific nature of the reduction of the normalized spectral power in the $15-45-\mathrm{Hz}$ band remains unclear. What is clear, however, is that the reduction in normalized spectral power around 300-600 ms indicates distinct differences in the processing of the stimulus classes and, although it may be speculated that the parameters relevant to this reduction reflect task specificity rather than only stimulus specificity, the precise functional role of these parameters still remains to be clarified in further investigations.

Another characteristic enhancement of the normalized spectral power was evident around $800-1200 \mathrm{~ms}$ in the $18.6-32.5-\mathrm{Hz}$ band. In the auditory condition, the enhancement of normalized spectral power was larger after the presentation of language stimuli whereas in the visual condition a larger enhancement of the normalized spectral power was obtained after presentation of non-language stimuli. Only for the auditory modality did this effect seem to be in accordance with the cell assembly model [3]. Concerning the visual modality, one might argue that subjects were still able to process non-language stimuli as linguistic elements after some simple mental rotation of the false font characters, yet this argument remains speculative. As the enhancement of normalized spectral power appears relatively late, it can hardly serve as an indicator for language perception processes. It may, however, reflect linguistic repair mechanisms. In general, a simple relationship between language perception and oscillatory brain dynamics can be excluded for the enhancement of normalized spectral power around $800-1200 \mathrm{~ms}$ in the 18.6$32.5-\mathrm{Hz}$ band. It is possible that this phenomenon is related to higher-order cognitive processing, such as repair mechanisms or even problem solving. On the other hand, one might argue that this late enhancement of normalized spectral power simply reflects a return of previously activated brain structures into a rest or idling state. In this way, Pfurtscheller [34] explained the event-related synchronization of EEG activity which is best obtained during the relaxed awake state, a comatose state, or during sleep.

In summary, the results of this study show that an enhancement of the normalized spectral power compared to a baseline level in the frequency band around $60 \mathrm{~Hz}$ and the latency range around $300 \mathrm{~ms}$ might be interpreted as a correlate of specific cognitive processing. In an experimental situation where simple lexical processing took place, the normalized spectral power was predominantly enhanced over temporooccipital brain regions of the language-dominant left hemisphere. In contrast, during the perception of visually and acoustically presented non-verbal stimuli, the enhancement of the normalized spectral power was largest over centroparietal areas of the right hemisphere. This result might be taken as evidence for the activation of those cortical cell assemblies responsible for language processing located for the most part in the left hemisphere, thus, confirming Braitenberg and Pulvermüller's [3] cell assembly theory of language processing. On the other hand, it might be taken as evidence for the activation of other cortical cell assemblies situated for the most part in the right hemisphere, thus, accounting for the processing of non-verbal information [11].

Changes of the normalized spectral power in other time/frequency bins indicated differences in the processing of stimulus classes not directly related to linguistic processing. The reduction in normalized spectral power $(15-45 \mathrm{~Hz})$ mirrored task specificity rather than stimulus specificity alone. The late enhancement of normalized spectral power (around $20 \mathrm{~Hz}$ ) is possibly related to a higher-order cognitive processing or may simply reflect the return of a previously activated brain structures into a resting or idling state.

\section{Acknowledgements}

The research was supported by grants from the Deutsche Forschungsgemeinschaft and the Alfried Krupp von Bohlen und Halbach foundation. We thank Drs. H. Schriefers, A. Mecklinger and F. Rösler for helpful comments, S. Hampson for technical support, and C. Robert and D. Charbonnier for editing.

\section{References}

[1] Aertsen, A., Erb, M., Palm, G. and Schütz, A., Coherent assembly dynamics in the cortex: multi-neuron recordings, network simulations and anatomical considerations. In C. Pantev et al. (Eds.), Oscillatory Event Related Brain Dynamics, Plenum, New York, 1994, pp. 59-83.

[2] Berger, H., Über das Elektrenzephalogramm des Menschen, Arch. Psychiatrie Nervenkrankh., 87 (1929) 527-550. 
[3] Braitenberg, V. and Pulvermüller, F., Entwurf einer neurologischen Theorie der Sprache, Naturwissenschaften, 79 (1992) 103-117.

[4] Bressler, S.L. and Freeman, W.J., Frequency analysis of olfactory system EEG in cat, rabbit and rat, Electroencephalogr. Clin. Neurophysiol., 50 (1980) 19-24.

[5] Broca, P., Sur la faculté du langage articulé, Bull. Soc. Anthropol. Paris (1865) 493-494.

[6] Cacioppo, J.T., Tassinary, L.G. and Fridlund, A.J., The skeletomotor system. In J.T. Cacioppo and L.G. Tassinary (Eds.), Principles of Psychophysiology. Physical, Social, and Inferential Elements, Cambridge University Press, Cambrigde, UK, 1990, pp. 325-384.

[7] Eckhorn, R., Bauer, R., Jordan, W., Brosch, S., Kruse, W., Munk, M. and Reitboeck, H.J., Coherent oscillations: a mechasnism of feature linking in the visual cortex? Biol. Cybern., 60 (1988) 121130.

[8] Eulitz, C., Elbert, T., Bartenstein, P., Weiller, C., Müller, S.P. and Pantev, C., Comparison of magnetic and metabolic brain activity during a verb generation task, NeuroReport, 6 (1994) 97-100.

[9] Fischler, I.S., Comprehending language with event-related potentials. In J.W. Rohrbaugh et al. (Eds.), Event-Related Brain Potentials: Basic Issues and Applications, Oxford University Press, New York, NY, 1990, pp. 165-177.

[10] Friederici, A.D., Pfeifer, E. and Hahne, A., Event-related brain potentials during natural speech processing: effects of semantic, morphological and syntactic violations, Cogn. Brain Res., 1 (1993) 183-192.

[11] Geffen, G., Bradshaw, J.C. and Nettleton, N.C., Hemispheric asymmetry: verbal and spatial encoding of visual stimuli, Exp. Psychol., 95 (1972) 25-31.

[12] Gerstein, G.L., Bedenbaugh, P. and Aertsen, A.M.H.J., Neuronal assemblies, IEEE Trans. Biomed. Eng., 36 (1989) 4-14.

[13] Gray, C.M., König, P., Engel, A.K. and Singer, W., Oscillatory responses in cat visual cortex exhibit inter-columnar synchronization which reflects global stimulus properties, Nature, 338 (1989) 334337.

[14] Hinke, R.M., Hu, X., Stillman, A.E., Kim, S.-G., Merkle, H., Salmi, R. and Ugurbil, K., Functional magnetic resonance imaging of Broca's area during internal speech, NeuroReport, 4 (1993) 675678.

[15] Hoke, M., Lehnertz, K., Pantev, C. and Lütkenhöner, B., Spatiotemporal aspects of synergetic processes in the auditory cortex as revealed by the magnetoencephalogram. In E. Basar and T.H. Bullock (Eds.), Dynamics of Cognitive and Sensory Processing in the Brain, 2, Springer, Berlin, Germany, 1989, pp. 84-105.

[16] Holcomb, P.J. and Neville, H.J., Auditory and visual semantic priming in lexical decision: a comparison using event-related brain potentials, Lang. Cogn. Process., 5 (1990) 281-312.

[17] Jürgens, E., Rösler, F., Henninghausen, E. and Heil, M., Stimulusinduced gamma oscillations: harmonics of alpha activity? NeuroReport, 6 (1995) 813-816.

[18] Kertez, A., Harlock, W. and Coates, R., Computer tomographic localization, lesion size, and prognosis in aphasia and non verbal impairment, Brain Lang., 8 (1979) 34-50.

[19] Klimesch, W., Pfurtscheller, G., Mohl, W. and Schimke, H., Eventrelated desynchronisation, ERD-mapping and hemispheric differences for words and numbers, Int. J. Psychophysiol., 8 (1990) 297-308.

[20] Kristeva-Feige, R., Feige, B., Makeig, S., Ross, B. and Elbert, T., Oscillatory brain activity during a motor task, NeuroReport, 4 (1993) 1291-1294.

[21] Kutas, M. and Hillyard, S.A., Reading senseless sentences: brain potentials reflect semantic incongruity, Science, 207 (1980) 203-205.

[22] Kutas, M. and van Petten, C., Event-related potential studies of language. In P.K. Ackles et al. (Eds.), Advances in Psychophysiology, Vol. 3, JAI, Greenwich, UK, 1988, pp. 139-187.

[23] Lopes da Silva, F.H., Neural mechanisms underlying brain waves: from neural membranes to networks, Electroencephalogr. Clin. Neurophysiol., 79 (1991) 81-93.

[24] Lund, E., Spliid, P.E., Andersen, E. and Bojsen-Moller, A., Vowel perception: a neuroradiological study of the perception of vowels in the human cortex, Brain Lang., 25 (1986) 191-211.

[25] Lutzenberger, W., Pulvermüller, F. and Birbaumer, N., Words and pseudowords elicit distinct patterns of $30-\mathrm{Hz}$ EEG responses in humans, Neurosci. Lett., 176 (1994) 115-118.

[26] Lutzenberger, W., Pulvermüller, F., Elbert, T. and Birbaumer, N., Visual stimulation alters local $40-\mathrm{Hz}$ responses in humans: an EEG-study, Neurosci. Lett., 183 (1995) 39-42.

[27] Makeig, S., Auditory event-related dynamics of the EEG spectrum and effects of exposure to tones, Electroencephalogr. Clin. Neurophysiol., 86 (1993) 283-293.

[28] Mazoyer, B.M., Tzourio, N., Frak, V., Syrota, A., Murayama, N., Levrier, O., Salamon, G., Dehaene, S., Cohen, L. and Mehler, J., The cortical representation of speech, J. Cogn. Neurosc., 5 (1993) 467-479.

[29] Müller, M., Bosch, J., Elbert, T., Kreiter, A., Valdes-Sosa, M.P. and Rockstroh, B., Visually induced gamma band responses in human EEG - a link to animal studies, Exp. Brain Res., in press.

[30] Neville, H.J., Corina, D., Bavelier, D., Clark, V.P., Jezzard, P., Prinster, A., Karni, A., Lalwani, A., Rauschecker, J. and Turner, R., Biological constraints and effects of experience on cortical organization for language: an MRI study of sentence processing in English and American sign language (ASL) by deaf and hearing subjects, Soc. Neurosci. Abstr., 20 (1994) 352.

[31] Neville, H.J., Mills, D.S. and Lawson, D.S., Fractionating language: different neural subsystems with different sensitive periods, Cerebral Cortex, 2 (1992) 244-258.

[32] Oldfield, R.C., The assessment and analysis of handedness: the Edinburgh inventory, Neuropsychologia, 9 (1971) 97-113.

[33] Petersen, S.E. and Fiez, J.A., The processing of single words studied with positron emission tomography, Annu. Rev. Neurosci., 16 (1993) 509-530.

[34] Pfurtscheller, G., Event-related synchronization (ERS): an electrophysiological correlate of cortical areas at rest, Electroencephalogr. Clin. Neurophysiol., 83 (1992) 62-69.

[35] Pfurtscheller, G. and Klimesch, W., Functional topography during a visuoverbal judgment task studied with event-related desynchronization mapping, J. Clin. Neurophysiol., 9 (1992) 120-131.

[36] Pfurtscheller, G. and Neuper, C., Simultaneous EEG $10 \mathrm{~Hz}$ desynchronisation and $40 \mathrm{~Hz}$ synchronisation during finger movements, NeuroReport, 3 (1992) 1057-1060.

[37] Pulvermüller, F., Constituents of a neurological theory of language, Concepts Neurosc., 3 (1992) 157-200.

[38] Pulvermüller, F., Preiß1, H., Eulitz, C., Pantev, C., Lutzenberger, W., Elbert, T. and Birbaumer, N., Brain rhythms, cell assemblies and cognition: evidence from the processing of words and pseudowords, Psycoloquy, 5 (48) (1994).

[39] Rasmussen, T. and Milner, B., The role of early left-Brain injury in determining lateralisation of cerebral speech functions. In S. Dimond and D. Blizzard (Eds.), Evolution and Lateralization of the Brain, New York Academy of Sciences, New York, NY, 1977.

[40] Reicher, G.M., Perceptual recognition as a function of meaningfulness of stimulus material, J. Exp. Psychol., 81 (1969) 275-280.

[41] Rösler, F., Heil, M. and Henninghausen, E., Distinct cortical activation patterns during long-term memory retrieval of verbal, spatial and colour information, J. Cogn. Neurosc., 7 (1995) 51-65.

[42] Salmelin, R. and Hari, R., Characterization of spontaneous MEG rhythms in healthy adults, Electroencephalogr. Clin. Neurophysiol., 91 (1994) 237-248.

[43] Sams, M., Aulanko, R., Hämäläinen, M., Hari, R., Lounasmaa, V., Lu, S. and Simola, J., Seeing speech: visual information from lip movements modifies activity in the human auditory cortex, Neurosci. Lett., 127 (1991) 141-145. 
[44] Singer, W., Synchronization of cortical activity and its putative role in information processing and learning, Annu. Rev. Physiol., 55 (1993) 349-374.

[45] Sperry, R., A modified concept of consciousness, Physiol. Rev., 76 (1969) 532.

[46] Steriade, M., Gloor, P., Llinas, R., Lopes da Silva, F. and Mesulam, M.M., Basic mechanisms of cerebral rhythmic activities, Electroencephalogr. Clin. Neurophysiol., 76 (1990) 481-508.

[47] Valdes-Sosa, M., Bobes, M.A., Sierra, C., Echevarria, M., Perez, L., Bosch, J. and Valdes-Sosa, P., Attentional effects on image-motion related potentials and spectral pertubations. In C. Pantev et al. (Eds.), Oscillatory Event-Related Brain Dynamics, Plenum, New York, NY, 1994, pp. 147-166.

[48] Wheeler, D.D., Processes in word recognition, Cogn. Psychol., 1 (1970) 59-80.

[49] Wikswo, J.P., Biomagnetic sources and their models. In S.J. Williamson et al. (Eds.), Advances in Biomagnetism, Plenum, New York, NY, 1989, pp. 1-18.

[50] Zaidel, E., Auditory vocabulary of the right hemisphere following brain bisection or hemidecortication, Cortex, 12 (1976) 191-211. 